\title{
Reação de germoplasma crioulo de feijoeiro (Phaseolus vulgaris L.) a Colletotrichum lindemuthianum
}

\author{
Luis A.M. Medeiros¹, Ricardo S. Balardin², Ivan F.D. Costa², Caroline A. Gulart² \& Giuvan Lenz² \\ ${ }^{1}$ Centro Federal de Educação Tecnológica São Vicente do Sul, 97420-000, São Vicente do Sul, RS, Brasil; ${ }^{2}$ Departamento de \\ Defesa Fitossanitária, Universidade Federal de Santa Maria, Centro de Ciências Rurais, 97105-900, Santa Maria, RS, Brasil
}

Autor para correspondência: Caroline A. Gulart, e-mail: carolgulart@yahoo.com.br

RESUMO

Nos anos de 2003 e 2004, na Universidade Federal de Santa Maria, foram avaliados 60 genótipos de Feijoeiro Comum (Phaseolus vulgaris), de origem andina e meso-americana, com o objetivo de caracterizar sua estrutura genética quanto à resistência a 12 raças fisiológicas de Colletotrichum lindemuthianum, no estádio juvenil $\left(\mathrm{V}_{1} / \mathrm{V}_{2}\right)$. As reações de cada genótipo foram avaliadas com base em uma escala de notas com valores de 1 a 9 a partir das quais foram calculados dois índices de resistência para cada genótipo avaliado e um índice de virulência para cada raça inoculada. A análise fenética dos dados de virulência foi obtida a partir da construção de uma matriz, considerando as reações incompatíveis e compatíveis. Maior variabilidade genética em relação à resistência a Colletotrichum lindemuthianum foi observada no germoplasma Andino do que aquela apresentada pelo germoplasma Meso-americano. Níveis de resistência que podem não ser do tipo raça-específica também foram observados.

Palavras-chave: resistência genética, genótipos andinos e meso-americanos, antracnose.

\section{ABSTRACT}

Reaction of germplasm of Common Bean to Colletotrichum lindemuthianum

Sixty genotypes of common bean (Phaseolus vulgaris) from the Meso-American and Andean gene pool were evaluated in 2003 and 2004 at Universidade Federal de Santa Maria, State of Rio Grande do Sul, Brazil. Host genetic structure was evaluated based on the reaction of the sixty genotypes, in the juvenile state $\left(\mathrm{V}_{1} / \mathrm{V}_{2}\right)$ to 12 physiological races of Colletotrichum lindemuthianum. The phenetic analysis was generated from virulence data considering incompatible and compatible reactions. Lower resistance index was observed. However, complex resistance was found in some genotypes. Higher genetic variability for resistance to Colletotrichum lindemuthianum was observed in the Andean than the Meso-American germplasm.

Keywords: genetic resistance, andean germplasm, meso-american germplasm, anthracnose.

\section{INTRODUÇÃO}

A antracnose do feijoeiro comum, causada pelo fungo Colletotrichum lindemuthianum (Sacc. \& Magnus) Briosi \& Cavara, é a doença foliar fúngica mais importante dessa cultura (Hubbeling, 1977; Pastor-Corrales \& Tu, 1989; Rava et al., 1993). Encontra-se amplamente disseminada nas regiões de cultivo, sendo favorecida por temperaturas do ar amenas e alta umidade relativa do ar (Chaves, 1980; Vieira, 1988). Em condições ambientais favoráveis, a doença pode provocar redução no rendimento da cultura de até $100 \%$, quando utilizadas sementes infectadas de cultivares suscetíveis (Zaumeyer \& Thomas, 1957). No Brasil, a antracnose é capaz de limitar significativamente a produção de feijão, principalmente nas regiões serranas de estados das regiões Sul e Sudeste, onde as condições são favoráveis ao seu desenvolvimento (Rava et al., 1994).

A antracnose do feijoeiro apresenta controle difícil, principalmente pelo fato do patógeno possuir eficiente transmissão através de sementes e grande capacidade de sobrevivência em restos culturais infectados nas regiões de clima temperado (Sutton, 1992; Tu, 1992). O controle químico da doença é significativamente oneroso e, como há dificuldades de implementação do manejo integrado de doenças (Pastor-Corrales \& Tu, 1989), as pesquisas têm sido direcionadas para a resistência genética por ser esta a estratégia mais eficiente e econômica de se controlar a doença.

A resistência do tipo vertical, baseada em um ou poucos genes, tem sido preferencialmente buscada devido à maior facilidade de ser identificada e incorporada. No entanto, a variabilidade patogênica de C. lindemuthianum tem limitado a sua utilização (Barrus, 1918; Andrus \& Wade, 1942; Waterhouse, 1975; Meneses \& Dianese, 1988; Balardin et al., 1990; Somavilla \& Prestes, 1999).

Genótipos de cultivo local (crioulos), que representam fontes potenciais de variabilidade genética do hospedeiro, ainda existem em número significativo, apesar de sua área de cultivo ser limitada, comparativamente à área de plantio com cultivares recomendados pela pesquisa (Balardin et $a l ., 1990)$. Isto resulta no fato de que a maior variabilidade genética do feijoeiro encontra-se em pequenas áreas, 
enquanto a maior parte das áreas cultivadas é representada por poucos cultivares, o que acarreta redução na diversidade da população do feijoeiro, em plantios comerciais. Tal fato aumenta a vulnerabilidade e, conseqüentemente, a durabilidade da resistência vertical presente em cultivares desenvolvidas pela pesquisa ou melhoradas (Robinson, 1976).

A obtenção de cultivares com uma base genética ampla pode implicar em maior durabilidade da resistência, dificultando o acúmulo de alelos de virulência no patógeno o que seria capaz de provocar um esgotamento da variabilidade genética no hospedeiro (Balardin, 1997). Estudos que permitam conhecer o arranjo da base genética da resistência, disponível do hospedeiro, em relação à variabilidade do patógeno, são fundamentais para o sucesso na obtenção de resistência durável. O presente trabalho teve por objetivo a caracterização da reação de germoplasma crioulo de feijoeiro comum a raças fisiológicas de $C$. lindemuthianum.

\section{MATERIAL E MÉTODOS}

Foram utilizados 27 cultivares crioulos de feijoeiro comum de origem andina e 33 meso-americanas, sendo a maioria procedente do meio-oeste do Estado de Santa Catarina. Tais cultivares foram inoculados com as raças fisiológicas de C. lindemuthianum 9, 15, 17, 23, 31, 65, $81,87,89,453,521$, e 2047, oriundas da micoteca do Departamento de Defesa Fitossanitária da Universidade Federal de Santa Maria. As raças foram selecionadas a partir da distância genética apresentada através dos dados fenotípicos e molecular, sendo este grupo, considerado representativo de uma porção geneticamente significativa da variabilidade do patógeno (Balardin, 1997).

O isolado de cada uma das raças fisiológicas de C. lindemuthianum oriundo de cultura monospórica foi multiplicado em meio de cultura Mathur (Mathur, 1950). O inóculo final foi obtido através da transferência de porções de meio contendo colônias desenvolvidas do fungo para Erlenmayers com meio de cultura constituído por vagens verdes de feijoeiro picadas e esterilizadas. Os frascos foram incubados a temperatura ambiente, durante 15 dias na ausência de luz, para a obtenção de abundante esporulação.

Sementes dos 60 genótipos de $P$. vulgaris foram semeadas em bandejas plásticas medindo $34 \mathrm{~cm} \mathrm{x} 23 \mathrm{~cm} \mathrm{x}$ $7 \mathrm{~cm}$, contendo substrato elaborado a partir da mistura (v/v/ v) de solo peneirado (35\%), cama de aves peneirada (30\%) e casca de arroz (35\%) na proporção de quatro cultivares por bandeja. Foram semeadas 10 a 12 sementes de cada cultivar que foram, em seguida, colocadas para germinar em estufa plástica com ambiente parcialmente controlado. Nesse ambiente há redução da temperatura do ar através da umidificação e renovação forçada por exaustores e sistema de elevação da umidade relativa do ar através de nebulização, ambos acionados automaticamente.

A suspensão de esporos para a inoculação foi preparada a partir da adição $100 \mathrm{~mL}$ de água destilada esterilizada mais $5 \mathrm{~mL}$ de solução de Tween $80(0,01 \%)$ nos Erlenmayers com crescimento do patógeno, seguindose agitação e posterior filtragem da suspensão. A suspensão de esporos obtida foi ajustada para a concentração de 1,2 x $10^{6}$ esporos/mL, por contagem em câmara de Neubauer. Oito a 12 plântulas no estádio $\mathrm{V}_{1} / \mathrm{V}_{2}$ de cada cultivar foram aspergidas completamente com suspensão de esporos de cada raça fisiológica. Uma barra de pulverização acoplada a um reservatório com capacidade de $2 \mathrm{~L}$ e um cilindro de dióxido de carbono foram utilizados para esse procedimento, sendo as plântulas mantidas em condições de umidade relativa do ar acima de $95 \%$ e temperatura do ar entre 22 e $27^{\circ} \mathrm{C}$ por 48 horas. Posteriormente as plântulas foram colocadas em estufa plástica onde permaneceram até a época de avaliação.

Sete dias após a inoculação, a reação dos genótipos foi avaliada de acordo com escala descritiva de 1 a 9 (Balardin et al., 1990). Plântulas sem sintomas visíveis nas folhas ou com poucos sintomas, diversas lesões de tamanho diminuto, principalmente nas folhas primárias foram classificadas como resistentes (graus 1 a 3). Plântulas com pequeno número de lesões de tamanho médio ou grande, numerosas lesões pequenas, numerosas lesões de tamanho médio, com cancros nas hastes ou nos pecíolos, ou em ambas as faces das folhas sem causar a morte, foram classificadas como intermediárias (graus 3,1 a 6,9). Plântulas com cancros nas hastes e/ou nos pecíolos e/ou em ambas as faces das folhas podendo evoluir até a morte da plântula, foram classificadas como suscetíveis (graus 7 a 9).

Foi utilizado delineamento experimental inteiramente casualizado. Os dados obtidos foram analisados considerando o índice de virulência (IV) das raças, índice de resistência total (IRt), índice de resistência Brasil (IRb), e análise fenética.

O índice de virulência de cada uma das raças de $C$. lindemuthianum foi computado com base na reação dos 60 genótipos, agrupados como cultivares crioulas andinas e meso-americanas, considerando o número de cultivares com reação de suscetibilidade, sendo calculado pela equação: $\mathrm{IV}=$ Genótipos suscetíveis/total de genótipos inoculados.

O índice de resistência de cada genótipo foi calculado pelas equações: IRt $=$ Reações de resistência/total de raças inoculadas $(9,15,17,23,31,65,81,87,89,453,521$, e 2047), IRb= Reações de resistência/total de raças inoculadas com ocorrência relatada no Brasil $(9,15,17,23,31,65,81$, 87, 89, 453). Para efeito do cálculo de IV, IRt e IRb, os genótipos de reação intermediária foram considerados como suscetíveis.

Os dados da análise fenética de virulência/ suscetibilidade foram obtidos a partir da matriz gerada pelos valores das reações, sendo considerado 0 para resistência e 1 para reação suscetível. A matriz de similaridade para virulência/suscetibilidade foi gerada através do programa SIMQUAL do Sistema de análises multivariadas e taxonomia numérica para computadores (NTSYS-pc) versão 1.70 (Exeter Software, Setauker, NY). As diferenças genéticas individuais entre as populações dos genótipos e das raças foram calculadas utilizando-se o coeficiente Jaccard. Os 
coeficientes de similaridade para agrupar os indivíduos foram calculados através do processo SAHN (NTSYS-pc) pelo método UPGMA. Os fenogramas foram gerados pelo programa TREE DISPLAY (NTSYS-pc).

\section{RESULTADOS E DISCUSSÃO}

A reação do germoplasma a 12 raças fisiológicas de $C$. lindemuthianum mostrou reduzido índice de resistência geral nesses genótipos (Tabelas 1 e 2). Vinte e cinco genótipos apresentaram IRb abaixo de $36,4 \%$ enquanto outros 27 mostraram IRb entre 36,4 e $63,6 \%$. Apenas quatro genótipos andinos e três meso-americanos mostraram resistência mínima a $80 \%$ das raças relatadas nas regiões produtoras brasileiras. Neste grupo, destacaram-se os andinos CF 36, CF 62 e CF 63 com IRb de 90,1\% e CF 31 com IRb de
$100 \%$; os meso-americanos CF 22 e CF 66 com IRb de $81,8 \%$ e o genótipo Vermelho, que foi resistente a todas as raças inoculadas (Tabelas 1 e 2).

Observação semelhante pode ser feita através da análise fenética que permitiu a identificação de dois grupos principais, tanto para o germoplasma andino quanto para o meso-americano, considerando-se as reações apresentadas ao conjunto de raças. O genótipo Vermelho apresentou maior divergência em relação ao grupo formado pelo restante dos genótipos meso-americanos (Figura 1), ao passo que os genótipos CF 36, CF 63, CF 62 e CF 31, muito similares entre si quanto às reações apresentadas, formaram grupo distinto daquele formado pelo restante dos genótipos de origem andina (Figura 2).

As reações apresentadas por $\mathrm{CF} 31$, resistente às raças $9,15,17,23,31,65,81,87,89,453$ e 521 (Tabela

TABELA 1 - Índices de virulência (IV) e de resistência (IR) obtidos a partir da reação de cultivares crioulas de origem andina a 12 raças de Colletotrichum lindemuthianum.

\begin{tabular}{|c|c|c|c|c|c|c|c|c|c|c|c|c|c|c|}
\hline \multirow[t]{2}{*}{ Germoplasma } & \multicolumn{12}{|c|}{ Raças fisiológicas $^{1}$} & \multirow[t]{2}{*}{ IRt $^{2}$} & \multirow[t]{2}{*}{$\mathbf{I R b}^{3}$} \\
\hline & 9 & 15 & 17 & 23 & 31 & 65 & 81 & 87 & 89 & 453 & 521 & 2047 & & \\
\hline CF 123 & $\mathrm{I}^{4}$ & $\mathrm{~S}^{5}$ & $\mathrm{~S}$ & $\mathrm{~S}$ & $\mathrm{~S}$ & $\mathrm{~S}$ & $\mathrm{~S}$ & $\mathrm{~S}$ & $\mathrm{~S}$ & $\mathrm{~S}$ & $\mathrm{~S}$ & $\mathrm{~S}$ & 0,0 & 0,0 \\
\hline CF 45 & S & I & I & S & S & I & S & S & $\mathrm{S}$ & I & I & I & $\mathbf{0 , 0}$ & $\mathbf{0 , 0}$ \\
\hline CF 69 CAIBI & S & S & S & S & S & S & I & S & S & S & S & S & 0,0 & 0,0 \\
\hline CF 73 & S & S & S & S & S & S & S & S & S & S & S & S & $\mathbf{0 , 0}$ & $\mathbf{0 , 0}$ \\
\hline INDIANO & S & S & S & S & S & S & S & S & S & S & S & I & $\mathbf{0 , 0}$ & $\mathbf{0 , 0}$ \\
\hline MOURO & S & S & $\mathrm{S}$ & S & S & S & S & S & S & S & S & S & $\mathbf{0 , 0}$ & $\mathbf{0 , 0}$ \\
\hline OURO & S & $\mathrm{S}$ & S & S & S & I & S & S & S & S & S & I & 0,0 & $\mathbf{0 , 0}$ \\
\hline PERDIZ & $\mathrm{S}$ & $\mathrm{S}$ & I & $\mathrm{S}$ & $\mathrm{S}$ & I & $\mathrm{S}$ & $\mathrm{S}$ & I & I & $\mathrm{R}$ & I & 8,3 & 9,1 \\
\hline CF 03 & $\mathrm{R}^{6}$ & $\mathrm{R}$ & S & S & S & $\mathrm{R}$ & S & S & S & I & $\mathrm{R}$ & I & 33,3 & 36,4 \\
\hline CF 11 & I & $\mathrm{R}$ & S & S & S & $\mathrm{R}$ & S & S & S & $\mathrm{R}$ & $\mathrm{R}$ & S & 33,3 & 36,4 \\
\hline CF 06 & $\mathrm{R}$ & $\mathrm{R}$ & S & S & $\mathrm{S}$ & $\mathrm{R}$ & $\mathrm{S}$ & $\mathrm{S}$ & I & $\mathrm{R}$ & $\mathrm{R}$ & I & 41,7 & 45,5 \\
\hline CF 12 & $\mathrm{R}$ & $\mathrm{R}$ & $\mathrm{S}$ & $\mathrm{S}$ & $\mathrm{S}$ & $\mathrm{R}$ & $\mathrm{S}$ & $\mathrm{S}$ & I & $\mathrm{R}$ & $\mathrm{R}$ & $\mathrm{S}$ & 41,7 & 45,5 \\
\hline CF 37 & $\mathrm{R}$ & $\mathrm{R}$ & S & S & S & $\mathrm{R}$ & S & S & $\mathrm{S}$ & $\mathrm{R}$ & $\mathrm{R}$ & I & 41,7 & 45,5 \\
\hline CF 38 & $\mathrm{R}$ & $\mathrm{R}$ & S & I & I & $\mathrm{R}$ & S & S & I & $\mathrm{R}$ & $\mathrm{R}$ & S & 41,7 & 45,5 \\
\hline CF 39 & I & $\mathrm{R}$ & S & S & S & $\mathrm{R}$ & $\mathrm{R}$ & S & I & $\mathrm{R}$ & $\mathrm{R}$ & S & 41,7 & 45,5 \\
\hline RESPINGO & I & $\mathrm{R}$ & I & S & S & $\mathrm{R}$ & $\mathrm{R}$ & S & $\mathrm{R}$ & S & $\mathrm{R}$ & I & 41,7 & 45,5 \\
\hline CF 65 & $\mathrm{R}$ & $\mathrm{R}$ & S & S & S & I & $\mathrm{R}$ & I & I & $\mathrm{R}$ & $\mathrm{R}$ & I & 41,7 & 45,5 \\
\hline CF 47 & $\mathrm{R}$ & $\mathrm{R}$ & I & $\mathrm{R}$ & I & $\mathrm{R}$ & I & I & I & $\mathrm{R}$ & $\mathrm{R}$ & I & $\mathbf{5 0 , 0}$ & 54,5 \\
\hline CF 53 & $\mathrm{R}$ & $\mathrm{R}$ & S & S & S & $\mathrm{R}$ & $\mathrm{R}$ & S & I & $\mathrm{R}$ & $\mathrm{R}$ & I & $\mathbf{5 0 , 0}$ & 54,5 \\
\hline CF 58 & $\mathrm{R}$ & $\mathrm{R}$ & $\mathrm{S}$ & $\mathrm{S}$ & $\mathrm{S}$ & $\mathrm{R}$ & $\mathrm{R}$ & $\mathrm{S}$ & I & $\mathrm{R}$ & $\mathrm{R}$ & $\mathrm{S}$ & 50,0 & 54,5 \\
\hline CF 64 & $\mathrm{R}$ & $\mathrm{R}$ & $\mathrm{R}$ & I & I & $\mathrm{R}$ & $\mathrm{S}$ & I & $\mathrm{R}$ & $\mathrm{R}$ & I & I & $\mathbf{5 0 , 0}$ & 54,5 \\
\hline CF 24 & $\mathrm{R}$ & $\mathrm{R}$ & S & S & S & $\mathrm{R}$ & $\mathrm{R}$ & S & $\mathrm{R}$ & $\mathrm{R}$ & $\mathrm{R}$ & S & 58,3 & 63,6 \\
\hline CF 30 & $\mathrm{R}$ & $\mathrm{R}$ & $\mathrm{R}$ & I & I & $\mathrm{R}$ & $\mathrm{R}$ & S & $\mathrm{R}$ & I & $\mathrm{R}$ & I & 58,3 & 63,6 \\
\hline CF 36 & $\mathrm{R}$ & $\mathrm{R}$ & $\mathrm{R}$ & $\mathrm{R}$ & $\mathrm{R}$ & $\mathrm{R}$ & S & $\mathrm{R}$ & $\mathrm{R}$ & $\mathrm{R}$ & $\mathrm{R}$ & I & 83,3 & 90,1 \\
\hline CF 62 & $\mathrm{R}$ & $\mathrm{R}$ & $\mathrm{R}$ & $\mathrm{R}$ & $\mathrm{R}$ & $\mathrm{R}$ & S & $\mathrm{R}$ & $\mathrm{R}$ & $\mathrm{R}$ & $\mathrm{R}$ & I & 83,3 & 90,1 \\
\hline CF 63 & $\mathrm{R}$ & $\mathrm{R}$ & $\mathrm{R}$ & $\mathrm{R}$ & $\mathrm{R}$ & $\mathrm{R}$ & S & $\mathrm{R}$ & $\mathrm{R}$ & $\mathrm{R}$ & $\mathrm{R}$ & I & 83,3 & 90,1 \\
\hline CF 31 & $\mathrm{R}$ & $\mathrm{R}$ & $\mathrm{R}$ & $\mathrm{R}$ & $\mathrm{R}$ & $\mathrm{R}$ & $\mathrm{R}$ & $\mathrm{R}$ & $\mathrm{R}$ & $\mathrm{R}$ & $\mathrm{R}$ & I & 91,7 & 100,0 \\
\hline IV $^{7}$ & 40,7 & 29,6 & 77,8 & 81,5 & 85,2 & 33,3 & 70,4 & 85,2 & 70,4 & 40,7 & 29,6 & 100,0 & & \\
\hline
\end{tabular}

${ }^{1}$ Raças identificadas de acordo com o sistema binário de nomenclatura (Pastor-Corrales, 1994). Raças designadas anteriormente por letras gregas: 17- alfa; 23- delta; 31- capa; 65- epsilon; 81- eta; 87- mu; 89- alfa-Brasil; 453- zeta (Balardin \& Kelly, 1997); ${ }^{2}$ IRtÍndice de resistência dos genótipos considerando todas as raças do estudo; ${ }^{3} \mathrm{IRb}$ - Índice de resistência dos genótipos, considerando as raças relatadas no Brasil $\left(9,15,17,23,31,65,81,87,89\right.$ e 453); ${ }^{4}$ - Reação intermediária; ${ }^{5} \mathrm{~S}$ - Reação Suscetível; ${ }^{6} \mathrm{R}$ - Reação resistente (Balardin et al., 1990); ${ }^{7}$ IV- Índice de virulência das raças. 
TABELA 2 - Índices de virulência (IV) e de resistência (IR) obtidos a partir da reação de cultivares crioulas de origem meso-americana a 12 raças de Colletotrichum lindemuthianum

\begin{tabular}{|c|c|c|c|c|c|c|c|c|c|c|c|c|c|c|}
\hline \multirow[t]{2}{*}{ Germoplasma } & \multicolumn{12}{|c|}{ Raças fisiológicas $^{1}$} & \multirow[t]{2}{*}{$\mathrm{IRt}^{2}$} & \multirow[t]{2}{*}{$\mathrm{IRb}^{3}$} \\
\hline & 9 & 15 & 17 & 23 & 31 & 65 & 81 & 87 & 89 & 453 & 521 & 2047 & & \\
\hline AMENDOIM & $\mathrm{S}$ & S & S & S & $\mathrm{S}$ & $\mathrm{S}$ & S & $\mathrm{S}$ & $\mathrm{S}$ & $\mathrm{S}$ & $\mathrm{S}$ & $\mathrm{S}$ & $\mathbf{0 , 0}$ & $\mathbf{0 , 0}$ \\
\hline CF 10 & S & $\mathrm{S}$ & $\mathrm{S}$ & S & $\mathrm{S}$ & $\mathrm{S}$ & $\mathrm{I}^{4}$ & $S^{5}$ & $\mathrm{~S}$ & $\mathrm{~S}$ & I & $\mathrm{S}$ & $\mathbf{0 , 0}$ & 0,0 \\
\hline CF 128 ABELARDO & S & $\mathrm{S}$ & $\mathrm{S}$ & S & $\mathrm{S}$ & $\mathrm{S}$ & I & $\mathrm{S}$ & $\mathrm{S}$ & $\mathrm{S}$ & I & $\mathrm{S}$ & $\mathbf{0 , 0}$ & $\mathbf{0 , 0}$ \\
\hline CF 14 & I & $\mathrm{S}$ & I & S & S & I & S & $\mathrm{S}$ & I & $\mathrm{S}$ & I & S & $\mathbf{0 , 0}$ & $\mathbf{0 , 0}$ \\
\hline CF 16 & S & $\mathrm{S}$ & S & S & S & $\mathrm{S}$ & S & I & S & $\mathrm{S}$ & I & $\mathrm{S}$ & $\mathbf{0 , 0}$ & $\mathbf{0 , 0}$ \\
\hline CF 17 & $\mathrm{~S}$ & $\mathrm{~S}$ & S & S & S & $\mathrm{S}$ & I & $\mathrm{S}$ & S & $\mathrm{S}$ & I & S & $\mathbf{0 , 0}$ & 0,0 \\
\hline CF 19 & S & S & S & S & S & $\mathrm{S}$ & S & I & $\mathrm{S}$ & $\mathrm{S}$ & S & $\mathrm{S}$ & $\mathbf{0 , 0}$ & $\mathbf{0 , 0}$ \\
\hline CF 25 & S & $\mathrm{S}$ & S & S & S & $\mathrm{S}$ & I & I & $\mathrm{S}$ & $\mathrm{S}$ & I & S & 0,0 & $\mathbf{0 , 0}$ \\
\hline $\mathrm{CF} 42$ & $\mathrm{~S}$ & $\mathrm{~S}$ & I & $\mathrm{S}$ & $\mathrm{S}$ & $\mathrm{S}$ & $\mathrm{S}$ & $\mathrm{S}$ & $\mathrm{S}$ & $\mathrm{S}$ & $\mathrm{S}$ & $\mathrm{S}$ & $\mathbf{0 , 0}$ & $\mathbf{0 , 0}$ \\
\hline $\mathrm{CF} 43$ & $\mathrm{~S}$ & $\mathrm{~S}$ & $\mathrm{~S}$ & $\mathrm{~S}$ & $\mathrm{~S}$ & $\mathrm{~S}$ & S & $\mathrm{S}$ & $\mathrm{S}$ & $\mathrm{S}$ & $\mathrm{S}$ & $\mathrm{S}$ & $\mathbf{0 , 0}$ & $\mathbf{0 , 0}$ \\
\hline CF 26 & S & S & S & S & S & $\mathrm{S}$ & $\mathrm{R}^{6}$ & $\mathrm{~S}$ & S & $\mathrm{S}$ & $\mathrm{S}$ & $\mathrm{S}$ & 8,3 & 9,1 \\
\hline CF 68 & $\mathrm{~S}$ & $\mathrm{~S}$ & I & S & S & $\mathrm{S}$ & $\mathrm{R}$ & S & S & S & I & S & 8,3 & 9,1 \\
\hline CF 70 CHAPECÓ & I & $\mathrm{S}$ & $\mathrm{S}$ & S & S & $\mathrm{R}$ & S & $\mathrm{S}$ & S & $\mathrm{S}$ & I & I & 8,3 & 9,1 \\
\hline CF 126 PALMITOS & I & I & S & I & S & I & S & $\mathrm{R}$ & I & I & $\mathrm{R}$ & $\mathrm{S}$ & 16,7 & 18,2 \\
\hline CF 71 & I & I & S & $\mathrm{S}$ & $\mathrm{S}$ & $\mathrm{R}$ & S & $\mathrm{S}$ & $\mathrm{S}$ & $\mathrm{S}$ & $\mathrm{R}$ & I & 16,7 & 18,2 \\
\hline SANTA TEREZINHA & I & $\mathrm{S}$ & I & $\mathrm{S}$ & S & $\mathrm{R}$ & S & $\mathrm{S}$ & I & $\mathrm{S}$ & $\mathrm{R}$ & $\mathrm{S}$ & 16,7 & 18,2 \\
\hline CF 49 & $\mathrm{R}$ & $\mathrm{R}$ & $\mathrm{S}$ & $\mathrm{S}$ & S & I & S & $\mathrm{S}$ & $\mathrm{S}$ & $\mathrm{S}$ & $\mathrm{R}$ & $\mathrm{S}$ & 25,0 & 27,3 \\
\hline CF 08 & I & I & $\mathrm{R}$ & I & I & $\mathrm{R}$ & S & $\mathrm{R}$ & I & $\mathrm{R}$ & I & I & 33,3 & 36,4 \\
\hline PRETO MANTEIGA & I & $\mathrm{R}$ & I & I & S & $\mathrm{R}$ & $\mathrm{R}$ & I & I & I & $\mathrm{R}$ & $\mathrm{S}$ & 33,3 & 36,4 \\
\hline CF 122 MISTURA & I & $\mathrm{R}$ & $\mathrm{R}$ & I & $\mathrm{R}$ & I & S & $\mathrm{R}$ & I & $\mathrm{S}$ & $\mathrm{R}$ & $\mathrm{S}$ & 41,7 & 45,4 \\
\hline CF 18 & I & $\mathrm{S}$ & $\mathrm{R}$ & $\mathrm{S}$ & $\mathrm{S}$ & $\mathrm{R}$ & S & $\mathrm{R}$ & $\mathrm{R}$ & $\mathrm{R}$ & I & $\mathrm{S}$ & 41,7 & 45,4 \\
\hline CF 51 & $\mathrm{R}$ & $\mathrm{R}$ & I & I & I & $\mathrm{R}$ & S & $\mathrm{R}$ & I & I & $\mathrm{R}$ & $\mathrm{S}$ & 41,7 & 45,4 \\
\hline CF 07 & $\mathrm{R}$ & I & $\mathrm{R}$ & $\mathrm{R}$ & I & $\mathrm{R}$ & I & I & $\mathrm{R}$ & $\mathrm{S}$ & $\mathrm{R}$ & $\mathrm{S}$ & $\mathbf{5 0 , 0}$ & 54,5 \\
\hline CF 09 & $\mathrm{R}$ & I & $\mathrm{R}$ & $\mathrm{R}$ & I & $\mathrm{R}$ & S & $\mathrm{R}$ & I & $\mathrm{R}$ & I & I & $\mathbf{5 0 , 0}$ & 54,5 \\
\hline CF 27 & $\mathrm{R}$ & I & $\mathrm{R}$ & I & I & $\mathrm{R}$ & I & I & $\mathrm{R}$ & $\mathrm{R}$ & I & $\mathrm{R}$ & $\mathbf{5 0 , 0}$ & 54,5 \\
\hline CF 52 & I & $\mathrm{R}$ & $\mathrm{R}$ & I & I & $\mathrm{R}$ & $\mathrm{R}$ & $\mathrm{R}$ & I & $\mathrm{R}$ & I & I & $\mathbf{5 0 , 0}$ & 54,5 \\
\hline CF 20 & $\mathrm{R}$ & $\mathrm{R}$ & $\mathrm{R}$ & $\mathrm{R}$ & I & $\mathrm{R}$ & S & $\mathrm{R}$ & I & I & $\mathrm{R}$ & $\mathrm{S}$ & 58,3 & 63,6 \\
\hline $\mathrm{CF} 40$ & $\mathrm{R}$ & $\mathrm{R}$ & $\mathrm{R}$ & $\mathrm{R}$ & $\mathrm{R}$ & $\mathrm{R}$ & S & I & $\mathrm{S}$ & $\mathrm{S}$ & $\mathrm{R}$ & $\mathrm{S}$ & 58,3 & 63,6 \\
\hline CF 72 & $\mathrm{R}$ & $\mathrm{R}$ & $\mathrm{R}$ & $\mathrm{R}$ & $\mathrm{R}$ & $\mathrm{R}$ & S & I & $\mathrm{S}$ & $\mathrm{S}$ & $\mathrm{R}$ & S & 58,3 & 63,6 \\
\hline CF 34 & $\mathrm{R}$ & $\mathrm{R}$ & $\mathrm{R}$ & $\mathrm{R}$ & $\mathrm{R}$ & $\mathrm{R}$ & $\mathrm{S}$ & $\mathrm{R}$ & I & $\mathrm{S}$ & $\mathrm{R}$ & $\mathrm{S}$ & 66,7 & 72,7 \\
\hline CF 22 & $\mathrm{R}$ & $\mathrm{R}$ & $\mathrm{R}$ & $\mathrm{R}$ & $\mathrm{R}$ & I & $\mathrm{S}$ & $\mathrm{R}$ & $\mathrm{R}$ & $\mathrm{R}$ & $\mathrm{R}$ & $\mathrm{S}$ & 75,0 & 81,8 \\
\hline CF 66 & $\mathrm{R}$ & $\mathrm{R}$ & $\mathrm{R}$ & $\mathrm{R}$ & $\mathrm{R}$ & $\mathrm{R}$ & $\mathrm{S}$ & $\mathrm{R}$ & $\mathrm{S}$ & $\mathrm{R}$ & $\mathrm{R}$ & $\mathrm{S}$ & 75,0 & 81,8 \\
\hline VERMELHO & $\mathrm{R}$ & $\mathrm{R}$ & $\mathrm{R}$ & $\mathrm{R}$ & $\mathrm{R}$ & $\mathrm{R}$ & $\mathrm{R}$ & $\mathrm{R}$ & $\mathrm{R}$ & $\mathrm{R}$ & $\mathrm{R}$ & $\mathrm{R}$ & 100,0 & 100,0 \\
\hline $\mathbf{I} \mathbf{V}^{7}$ & 63,6 & 63,6 & 57,6 & 72,7 & 78,8 & 48,5 & 84,8 & 63,6 & 84,8 & $\mathbf{7 5 , 7}$ & $\mathbf{5 4 , 5}$ & 94,0 & & \\
\hline
\end{tabular}

${ }^{1}$ Raças identificadas de acordo com o sistema binário de nomenclatura (Pastor-Corrales, 1994). Raças designadas anteriormente por letras gregas: 17- alfa; 23- delta; 31- capa; 65- epsilon; 81- eta; 87- mu; 89- alfa-Brasil; 453- zeta (Balardin \& Kelly, 1997); ${ }^{2}$ IRt- Índice de resistência dos genótipos considerando todas as raças do estudo; ${ }^{3} \mathrm{IRb}$ - Índice de resistência dos genótipos, considerando as raças relatadas no Brasil $\left(9,15,17,23,31,65,81,87,89\right.$ e 453); ${ }^{4}$ I - Reação intermediária; ${ }^{5}$ S - Reação Suscetível; ${ }^{6}$ R Reação resistente (Balardin et al., 1990); ${ }^{7}$ IV- Índice de virulência das raças.

1) e Vermelho, resistente a todas as raças do estudo (Tabela 2) sugerem a existência de um tipo de resistência complexa nesses genótipos. Essa resistência, provavelmente estaria sendo determinada por mais de um gene a exemplo do que ocorre com os cultivares diferenciadores AB 136 com valor binário 1024 (Pastor-Corrales, 1991) e que possui os genes 

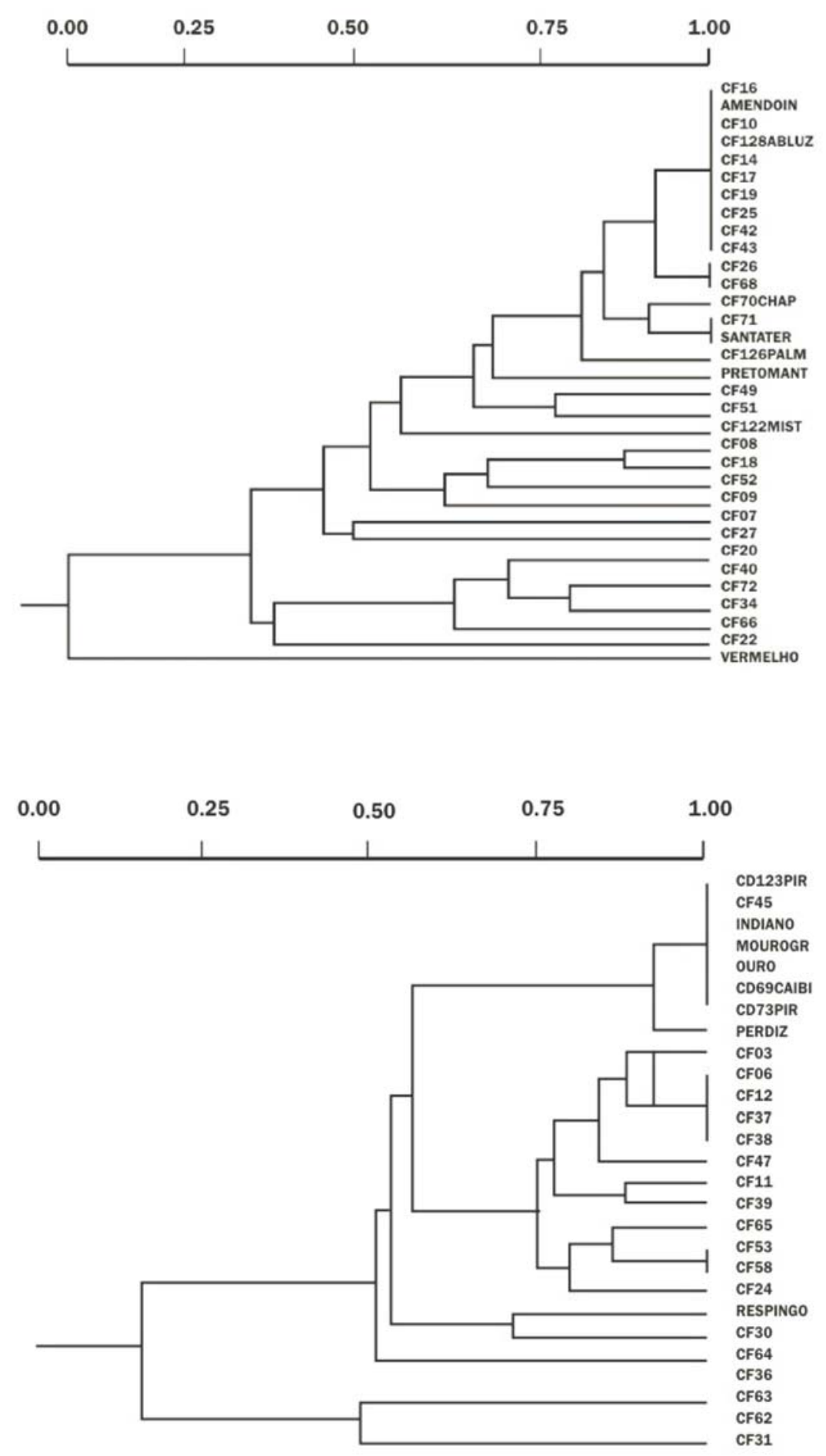

FIG. 1 - Fenograma de 33 genótipos crioulos meso-americanos de feijoeiro comum (Phaseolus vulgaris L.) baseado nos dados de virulência obtidos através da inoculação de 12 raças de Colletotrichum lindemuthianum.

FIG. 2 - Fenograma de 27 genótipos crioulos andinos de feijoeiro comum (Phaseolus vulgaris $\mathrm{L}$.) baseado nos dados de virulência obtidos através da inoculação de 12 raças de Colletotrichum lindemuthianum.
Co-6 e Co-8 (Young \& Kelly, 1996; Alzate-Marin et al., 2001) e G 2333 com valor binário 2048 (Pastor-Corrales, 1991), onde estão presentes os genes $\mathrm{Co}-4, \mathrm{Co}-5$ e $\mathrm{Co}$ 7 (Pastor-Corrales, 1994; Young \& Kelly, 1996; Young et al., 1998). Esses resultados podem ser considerados como evidência da existência de fontes genéticas capazes de conferir resistência complexa à antracnose do feijoeiro, a partir de germoplasma crioulo.

Maior amplitude de variação no IV das raças foi determinada pelo grupo de genótipos andinos (29,6\% a $100 \%$ ) em relação à observada através das reações dos genótipos meso-americanos (48,5\% a 94\%) (Tabelas 1 e 2). 
Esses resultados demonstram maior variabilidade de reação ao C. lindemuthianum presente no germoplasma andino do que aquela apresentada pelo germoplasma meso-americano.

Observação semelhante também foi obtida através do agrupamento das raças determinado pela análise fenética a partir da virulência apresentada ao germoplasma andino (Figura 3) e meso-americano (Figura 4). No agrupamento das raças determinado pelo germoplasma andino observa-se um grupo formado pelas raças 9, 453, 15, 65 e 521, com IV entre $29,6 \%$ e $40,7 \%$, distinto do outro grupo formado pelas raças $17,89,23,31,87,2047$ e 81 , com IV acima de 70\% (Tabela 1 e Figura 3). As raças dentro deste grupo possuem em comum a virulência a cultivar Widusa, valor binário 16 na série diferencial (Pastor-Corrales, 1991). No agrupamento das raças determinado pelo germoplasma meso-americano, apenas a raça 65 é divergente das demais, caracterizando menor variabilidade neste grupo, relacionada à resistência a C. lindemuthianum (Figura 4).

Entre as raças de ocorrência relatada no Brasil e que foram virulentas ao grupo de genótipos que apresentou IRb mínimo de $80 \%$ pode-se observar que a raça 81 foi virulenta a três cultivares andinos (CF 36, CF 62 e CF 63) e, associada às raças 65 e 89 venceu também a resistência de dois cultivares meso-americanos (CF 22 e CF 66) (Tabelas 1 e 2). Resultados semelhantes também foram obtidos quando da inoculação desta raça em genótipos comerciais e linhagens de feijoeiro
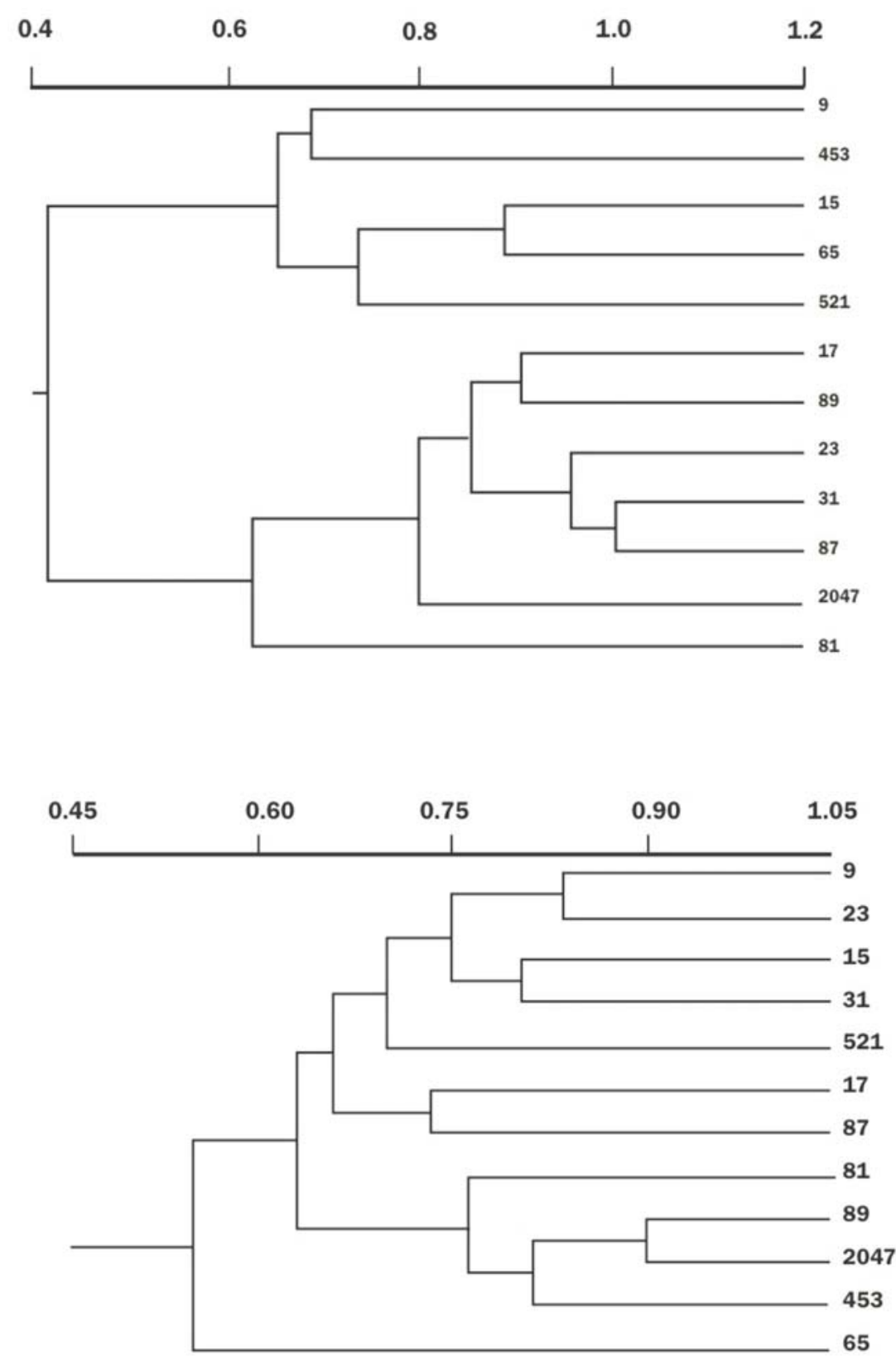

FIG. 3 - Fenograma de 12 raças fisiológicas de Colletotrichum lindemuthianum baseado nos dados de virulência obtidos através da inoculação de 27 genótipos crioulos andinos de feijoeiro comum (Phaseolus vulgaris).

FIG. 4 - Fenograma de 12 raças fisiológicas de Colletotrichum lindemuthianum baseado nos dados de virulência obtidos através da inoculação de 33 genótipos crioulos mesoamericanos de feijoeiro comum (Phaseolus vulgaris). 
comum. O estudo demonstrou a patogenicidade da raça 81 a duas entre oito das melhores linhagens e as três cultivares comerciais que apresentaram maior índice de resistência (Medeiros, 2004). Suscetibilidade à raça 81 também foi demonstrada para oito entre 14 cultivares recomendadas para o Estado de Minas Gerais, onde esta raça é considerada de ocorrência freqüente nas regiões produtoras (Lanza et al., 1997). Essa informação é importante por chamar a atenção para a necessidade de se verificar a frequência da raça 81 nas regiões de cultivo, quando da possível utilização destes genótipos em programa de incorporação de resistência à antracnose. Melhor compreensão sobre a estrutura populacional de $C$. lindemuthianum tem sido sugerida como estratégia fundamental para a incorporação de resistência durável à antracnose do feijoeiro (Balardin \& Kelly, 1998; Mahuku et al., 2002).

Os genótipos andinos CF 31, CF 36, CF 62 e CF 63, e os meso-americanos CF 22, CF 66 e Vermelho mostraramse materiais promissores para utilização em programas de melhoramento genético de feijoeiro, tendo apresentado índice de resistência (IRt e IRb) superior a 75\%.

Com base nos critérios de definição das classes de reação os genótipos CF 47, CF27 e CF52 não foram suscetíveis a nenhuma das raças inoculadas, apresentando reação de resistência à metade das raças inoculadas e intermediária às restantes (Tabelas 1 e 2). Estes dados indicam que apenas estes genótipos poderiam exibir resistência durável, por apresentarem tanto resistência vertical como resistência vertical incompleta, considerando a totalidade das raças testadas no trabalho. Outro fator relevante é o fato da raça 2047 não ter apresentado virulência sobre os mesmos, o que sugere uma base genética ampla capaz de manter-se durável mesmo que a população do patógeno possa sofrer alterações significativas, já que esta raça ataca todos os genes descritos no conjunto diferenciador utilizado e que contem genes utilizados nos programas de melhoramento de feijão.

\section{REFERÊNCIAS BIBLIOGRÁFICAS}

Alzate-Marin, AL, Souza KA, Barros EG, Moreira, MA (2001) Preliminary results of allelism studies for anthracnose resistance genes of common bean cultivar PI 207.262. Annual report of the Bean Improvement Cooperative Michigan 44:113-114. (Abstract)

Andrus CF, Wade BL (1942) The factorial interpretation of anthracnose resistance in beans. USDA Technical Bulletin 310:129.

Balardin RS, Pastor-Corrales MA, Otoya MM (1990) Variabilidade patogênica de Colletotrichum lindemuthianum no Estado de Santa Catarina. Fitopatologia Brasileira 15:243-245.

Balardin RS (1997) Identificação de raças fisiológicas de Colletotrichum lindemuthianum no Rio Grande do Sul. Fitopatologia Brasileira 27:50-53.

Balardin RS, Kelly JD (1997) Re characterization of Colletotrichum lindemuthianum races. Annual Report of the Bean Improvement Cooperative 40:126-127.

Balardin RS, Kelly JD (1998) Interaction between Colletotrichum lindemuthianum races and gene pool diversity in Phaseolus vulgaris. Journal of the American Society for Horticultural Science 123:1038-1047.

Barrus MF (1918) Varietal susceptibility of beans to strains of Colletotrichum lindemuthianum (Sacc. \& Magn.) B. \& C. Phytopathology 8:589-605.

Chaves G (2005) La antracnosis In: Schwartz HF, Galvez GE (Eds.) Problemas de Producción del frijol. Cali. CIAT. pp. 37-53.

Hubbeling N (1977) The new iota race of Colletotrichum lindemuthianum. Annual Report Bean Improvement Cooperative 20:58.

Lanza MA, Paula JR, Barros EG, Moreira MA (1997) Avaliação da resistência de cultivares de feijão recomendados para Minas Gerais à murcha de Fusarium. Fitopatologia Brasileira 22:274.

Mahuku GS, Jara C, Cuasquer JB, Castellanos G (2002) Genetic variability within Phaeoisariopsis griseola from Central America and its implications for resistance breeding of Common Bean. Plant Pathology 51:594-604.

Mathur RS, Barnett HL, Lilly VG (1950) Sporulation of Colletotrichum lindemuthianum in culture. Phytopathology 40:104-114.

Medeiros LAM (2004) Resistência genética do feijão (Phaseolus vulgaris L.) a Colletotrichum lindemuthianum. Tese de Doutorado. Santa Maria RS. Universidade Federal de Santa Maria.

Meneses JR, Dianese JC (1988) Race characterization of Brazilian isolates of Colletotrichum lindemuthianum and detection of resistance to anthracnose in Phaseolus vulgaris. Phytopathology 78:650-655

Pastor-Corrales MA, Tu JC (1989) Anthracnose. In: Schwartz HF, Pastor-Corrales MA (Eds.) Bean production problems in the tropics. Cali. CIAT. pp. 77-104.

Pastor-Corrales MA (1991) Estandarización de variedades diferenciales y de designación de razas de Colletotrichum lindemuthianum. Phytopathology 81:694. (Abstract)

Pastor-Corrales MA, Erazo OA, Estrada EI, Singh SP (1994) Inheritance of anthracnose resistance in common bean accession G2333. Plant Disease 78:959-962.

Rava CA, Molina J, Kauffmann M, Briones I (1993) Determinación de razas fisiológicas de Colletotrichum lindemuthianum en Nicaragua. Fitopatologia Brasileira 18:388-391.

Rava CA, Purchio AF, Sartorato A (1994) Caracterização de patótipos de Colletotrichum lindemuthianum que ocorrem em algumas regiões produtoras de feijoeiro comum. Fitopatologia Brasileira 19:167-172.

Robinson RA (1976) Plant pathosystems. Berlin. Springer Verlag.

Somavilla LL, Prestes AM (1999) Identificação de patótipos de Colletotrichum lindemuthianum de algumas regiões produtoras de feijão do Rio Grande do Sul. Fitopatologia Brasileira 24:416-421.

Sutton BC (1992) The Genus Glomerella and its anamorph Colletotrichum. In: Bailey JA, Jeger MJ (Eds.) Colletotrichum: biology, pathology and control. Wallingford. CAB International. pp. 1-26. 
Tu JC (1992) Colletotrichum lindemuthianum on bean. Population dynamics of the pathogen and breeding for resistance. In: Bailey JA, Jeger MJ (Eds.) Colletotrichum: biology, pathology and control. Wallingford. CAB. International. pp. 203-224.

Vieira C (1988) Doenças e Pragas do Feijoeiro. 2nd. Ed. Viçosa MG. Imprensa Universitária.

Waterhouse WL (1975) Studies of bean anthracnose in Australia. Proceedings of the Linnean Society of New South Wales 80:7183.
Young RA, Kelly JD (1996) Characterization of the genetic resistance to Colletotrichum lindemuthianum in common bean differential cultivars. Plant Disease 80:650-654.

Young RA, Melotto M, Nodari RO, Kelly JD (1998) Marker assisted dissection of oligogenic anthracnose resistance in the common bean cultivar, G 2333. Theoretical and Applied Genetics 96:87-94.

Zaumeyer WJ, Thomas HR (1957) A monografic study of bean diseases and methods for their control. Washington. USDA.

Recebido 24 Janeiro 2008 - Aceito 30 Junho 2008 - TPP 8008 Editor Associado: Carlos R. Casela 